\title{
The Impact of Chemotherapy on EGFR Mutation Status in Non-Small-Cell Lung Cancer: A Meta-Analysis
}

\author{
Xiaoshun Shi ${ }^{*}$, Fuxi Huang2*, Allen M. Chen ${ }^{3,4}$, Zhuolin Wu', Qianqian Huang6, Ying Liang1, \\ Qipeng Zhou ${ }^{7}$, Haiyun Mos, Xiaoxiang Li ${ }^{1}$, Jiexia Zhang1†
}

${ }^{1}$ National Clinical Research Center for Respiratory Disease, State Key Laboratory of Respiratory Disease, Department of Medicine, Guangzhou Institute of Respiratory Disease, The First Affiliated Hospital of Guangzhou Medical University, Guangzhou, China ${ }^{2}$ Department of Oncology, Panyu Central Hospital, Cancer Institute of Panyu, Guangzhou, China

${ }^{3}$ Department of Mathematics, University of California, Berkeley, USA

${ }^{4}$ Mendel Genes Inc, Manhattan Beach, USA

${ }^{5}$ Department of Biomedical Engineering, University of Minnesota, Twin Cities, USA

${ }^{6}$ Department of Hospital Infection Control, Affiliated Cancer Hospital \& Institute of Guangzhou Medical University, Guangzhou, China

${ }^{7}$ Department of Respiratory Medicine, Shantou Central Hospital, Shantou, China

${ }^{8}$ Department of Health Care, Maternal and Child Health Hospital of Haizhu District, Guangzhou, China

Email: ${ }^{\dagger} d r \_z h a n g j x @ 126 . c o m$

How to cite this paper: Shi, X.S., Huang, F.X., Chen, A.M., Wu, Z.L., Huang, Q.Q., Liang, Y., Zhou, Q.P., Mo, H.Y., Li, X.X. and Zhang, J.X. (2017) The Impact of Chemotherapy on EGFR Mutation Status in Non-Small-Cell Lung Cancer: A Meta-Analysis. Open Journal of Genetics, 7, 117-129.

https://doi.org/10.4236/ojgen.2017.74010

Received: October 11, 2017

Accepted: December 17, 2017

Published: December 21, 2017

Copyright $\odot 2017$ by authors and Scientific Research Publishing Inc. This work is licensed under the Creative Commons Attribution International License (CC BY 4.0).

http://creativecommons.org/licenses/by/4.0/

\begin{abstract}
Background: Emerging evidence indicates that chemotherapy for lung cancer may alter EGFR mutation status. However, whether chemotherapy as a firstline treatment may increase or reduce the frequency of EGFR mutations in NSCLC remains uncertain. Therefore, we conducted a meta-analysis to evaluate whether chemotherapy leads to altered EGFR mutation status. Methods: A systematic literature search was performed using the PubMed, OVID, Science Direct, Cochrane Library, and CNKI databases for studies on pre- and post-chemotherapy EGFR mutation status. Relevant studies documenting perichemotherapy EGFR mutation ratios were included. Analyses of pooled odds ratios (OR) were performed. Results: Six studies involving 656 patients were included in this meta-analysis. It was found that chemotherapy may alter EGFR status $(\mathrm{OR}=1.93,95 \%$ CI $1.05-3.56 ; \mathrm{p}<0.0001)$. No significant differences in EGFR mutation alterations were observed in terms of gender, smoking history, EGFR loci, or chemotherapy response in NSCLC patients. Conclusions: Chemotherapy may contribute to altered EGFR status. NSCLC
\end{abstract}

*Xiaoshun Shi and Fuxi Huang have contributed equally to this work and should be regarded as joint first authors. 
patients with EGFR mutations might need to be considered for EGFR status redeterminations prior to second-line EGFR-TKI treatment or upon tumor recurrence after chemotherapy. Further randomized clinical trials should investigate the impact of neoadjuvant or first-line chemotherapy on EGFR mutation status in NSCLC patients.

\section{Keywords}

EGFR Mutation, Chemotherapy, Non-Small Cell Lung Cancer, Meta-Analysis

\section{Background}

Lung cancer causes the majority of cancer deaths in China and worldwide [1]. Sensitive mutations of the epidermal growth factor receptor (EGFR) have been proven to be highly responsive to EGFR tyrosine kinase inhibitors (EGFR-TKIs) compared to standard chemotherapy [2] [3] in non-small cell lung cancer (NSCLC). However, EGFR-TKIs such as gefitinib or erlotinib are sometimes reserved for second-line or maintenance therapy in clinical practice. The somatic mutation status of the EGFR gene is a crucial biomarker for evaluating the application of EGFR-TKI treatment in patients with metastatic and chemo-resistant NSCLC.

Recent studies have investigated whether EGFR mutations may be altered by chemotherapy. The efficacy of EGFR-TKIs has been shown to be less potent when used as second-line treatment for NSCLC patients refractory to or intolerant of platinum-based combination chemotherapy [4]. On the other hand, some clinical studies have demonstrated increased serum EGFR mutation detection [5] [6]. In 2012, Honda et al. [7] reported a Japanese woman with an initial EGFR mutation (L747-T751 deletion in exon 19) that disappeared after chemotherapy. The same year, an oncological study by Bai et al. reported that chemotherapy may reduce the frequency of EGFR mutations in NSCLC patients [8]. Since then, the consideration of altered EGFR mutation status before and after chemotherapy has been tightly linked to the second-line EGFR-TKI treatment of NSCLC, prompting investigations by oncologists into the underlining mechanisms.

The aim of the present study was to systemically combine data from published articles to evaluate the impact of chemotherapy on EGFR mutation status in NSCLC. This meta-analysis is the first attempt to assess the role of chemotherapy in EGFR mutation alterations after standard chemotherapy, using evidencebased methods.

\section{Methods}

\subsection{Literature Search}

Relevant studies in English and Chinese were extracted from the PubMed, OVID, Science Direct, Cochrane Library, and China National Knowledge Infra- 
structure (CNKI) databases using the following terms: "non-small cell lung cancer", "NSCLC", "carcinoma", "neoplasm", "EGFR mutation", and "chemotherapy". The final search was conducted on June 24, 2016. All non-English and non-Chinese articles were excluded. To identify possible outcomes from unpublished trials containing potentially useful data, we also reviewed abstract books and presentations from major recent meetings of the American Society of Clinical Oncology (ASCO), the European Society for Medical Oncology (ESMO), and the World Conference on Lung Cancer. When the same patients were included in different publications, the most recent study was selected.

\subsection{Selection Criteria}

Eligible studies were gathered based on the following inclusion criteria: 1) standard chemotherapy as first-line treatment in chemo-naïve or radio-naïve NSCLC patients, 2) EGFR mutation status before and after chemotherapy, 3) any EGFR mutation subtype, 4) standard chemotherapy defined as platinumbased third-generation doublets, and 5) publication in English or Chinese. The exclusion criteria were: 1) duplicate reports, conference abstracts or papers, editorials, or reviews and 2) studies with insufficient data for pooled analysis.

\subsection{Data Extraction}

To identify all eligible research, two investigators (Xiaoshun Shi and Haiyun Mo) independently extracted the data and reached a consensus based on the inclusion criteria. The quality of each study was assessed using the Newcastle Ottawa Quality Assessment Scale. Cases of disagreement were resolved by discussion with a third author. The following information was extracted from the eligible studies: first author's surname, publication year, geographic region, chemotherapy agents used, EGFR detection method, and number of EGFR mutations before and after chemotherapy.

\subsection{Statistical Analysis}

Analysis for the forest plot was conducted using Review Manager 5.2 (Cochrane Collaboration, Oxford, UK). For predictable heterogeneity, pooled odds ratios (ORs) were calculated for all included parameters and presented by random effect models, as this generates wider confidence intervals and minimizes the risk of Type I errors. Heterogeneity among studies was evaluated with Cochran's $\mathrm{Q}$ test and the $\mathrm{I}^{2}$ statistic. For the subgroup analysis with high heterogeneity, we used both the random-effect model and the fixed-effect model to examine the final conclusion. Publication bias was assessed by Egger's test. The analysis of publication bias was done with STATA version 12.0. Additionally, the trim-and-fill method was used to adjust the risk estimates when the tests for publication bias were statistically significant [20], which were also analyzed by using STATA version 12.0. P-values of $<0.05$ were considered statistically significant. 


\section{Results}

\subsection{Study Description}

A total of 4983 articles were identified in the initial literature search, of which 4970 were rejected based on the exclusion criteria. Two articles were excluded because they belonged to conference abstracts and were subgroups in one of the included articles [8]. Studies with duplicate results, those not documenting EGFR mutation status in the peri-chemotherapy period, and those containing insufficient data for pooled analysis were also excluded. Finally, six articles were identified as eligible for this meta-analysis (Table 1 ). The total number of patients evaluated before and after chemotherapy was 656. All NSCLC stages were accepted in this meta-analysis. All patients included in the analysis were chemonaïve or radio-naïve prior to the detection of EGFR mutation status. ORs were extracted or calculated from each study (Figure 1).

Table 1. Characteristics of included prospective studies for meta-analysis.

\begin{tabular}{|c|c|c|c|c|c|c|c|c|c|c|c|}
\hline \multirow{2}{*}{ Author } & \multirow{2}{*}{ Year } & \multirow{2}{*}{ Region } & \multirow{2}{*}{ Study category } & \multirow{2}{*}{ Reagents } & \multirow{2}{*}{ Samples } & \multirow{2}{*}{ Method } & \multicolumn{2}{|c|}{ Pre-chemo } & \multicolumn{2}{|c|}{ Post-chemo } & \multirow{2}{*}{ - Ref. } \\
\hline & & & & & & & Mutation & Total & Mutation & Total & \\
\hline \multirow{2}{*}{ Munfus-McCray D } & \multirow{2}{*}{2013} & \multirow{2}{*}{ US } & \multirow{2}{*}{ Retrospective } & \multirow{2}{*}{$\begin{array}{l}\text { Platinum-based third-generation } \\
\text { doublets }\end{array}$} & \multirow{2}{*}{ Tissue } & \multirow{2}{*}{ PCR } & 31 & 37 & 6 & 37 & \multirow{2}{*}{ [9] } \\
\hline & & & & & & & 17 & 26 & 9 & 26 & \\
\hline \multirow{2}{*}{ Hua Bai } & \multirow{2}{*}{2012} & \multirow{2}{*}{ China } & \multirow{2}{*}{ Retrospective } & Cisplatin and carboplatin + & Blood & DHPLC & 91 & 264 & 61 & 264 & \multirow{2}{*}{ [8] } \\
\hline & & & & gemcitabine, vinorelbine and taxanes & Tissue & ARMS & 22 & 63 & 12 & 63 & \\
\hline Rubing HAN & 2011 & China & Retrospective & $\begin{array}{c}\text { Cisplatin/arboplatin }+ \\
\text { gemcitabine/pemetrexed/VP-16/taxanes }\end{array}$ & Serum & $\begin{array}{l}\text { Nested } \\
\text { PCR }\end{array}$ & 13 & 33 & 18 & 33 & {$[10]$} \\
\hline Hong-Gang Ke & 2013 & China & Retrospective & Pemetrexed + cisplatin & Tissue & PCR & 61 & 107 & 70 & 107 & {$[11]$} \\
\hline Yan-E Ma & 2013 & China & Retrospective & $\begin{array}{l}\text { Cisplatin/carboplatin }+ \\
\text { gemcitabine/vinorelbine/pemetrexed }\end{array}$ & Plasma & DHPLC & 18 & 60 & 15 & 60 & {$[12]$} \\
\hline
\end{tabular}

Records identified through datebase searching $(n=4983)$
Excluded 4972 articles (1) review articles, comments and editorials, Letter to editor, case reports, expert opinions, protocol, guidelines(2) animal experiment (3) Article did not document EGFR mutation status in after chemotherapy
Full-text retrieved for eligibility $(n=11)$

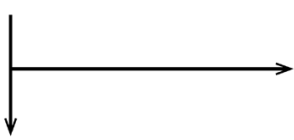

Studies include in this meta-analysis $(n=6)$
Excluded by details: (1)duplicate reports, conference abstracts or papers, editorials and reviews; (2)articles did not document EGFR mutation status in perichemotherapy period; (3) studies with insufficent data for pooled analysis

Figure 1. Flow diagram of the meta-analysis. 


\subsection{Effect of Chemotherapy on EGFR Mutation Status in NSCLC Patients}

Six studies were analyzed to examine the impact of chemotherapy on EGFR mutation status. Standard chemotherapy was defined as platinum-based third-generation doublets. The inconsistency index was $81 \%(\mathrm{p}<0.0001)$; therefore, a randomeffect model was applied. The result suggested that chemotherapy is a potential risk factor for the alteration of EGFR mutation status in NSCLC patients (OR = 1.93, 95\% CI 1.05 - 3.56; $\mathrm{p}<0.0001$ ) (Figure 2).

\subsection{Impact of Chemotherapy on EGFR Mutations with Regard to Clinicopathological Characteristics}

Four studies examined the impact of chemotherapy on EGFR alterations based on gender (Figure 3) and smoking status (Figure 4). The impact of chemothe-

\begin{tabular}{|c|c|c|c|c|c|c|c|c|c|c|}
\hline Study or Subgroup & \multicolumn{2}{|c|}{ Experimental } & \multicolumn{2}{|c|}{ Control } & Weight & $\begin{array}{l}\text { Odds Ratio } \\
\text { M-H, Random, } 95 \% \mathrm{Cl}\end{array}$ & \multicolumn{4}{|c|}{$\begin{array}{c}\text { Odds Ratio } \\
\mathrm{M}-\mathrm{H}, \text { Random, } 95 \% \mathrm{Cl}\end{array}$} \\
\hline 2011 Han & 13 & 33 & 18 & 33 & $11.6 \%$ & $0.54[0.20,1.44]$ & & & & \\
\hline 2012 Bai (1) & 91 & 264 & 61 & 264 & $15.4 \%$ & $1.75[1.19,2.57]$ & & & & \\
\hline 2012 Bai(2) & 22 & 63 & 12 & 63 & $12.7 \%$ & $2.28[1.01,5.15]$ & & & & \\
\hline $2013 \mathrm{Ke}$ & 61 & 107 & 70 & 107 & $14.5 \%$ & $0.70[0.40,1.22]$ & & & & \\
\hline $2013 \mathrm{Ma}$ & 18 & 60 & 15 & 60 & $12.8 \%$ & $1.29[0.58,2.87]$ & & & & \\
\hline 2013 Munfus (1) & 31 & 37 & 6 & 37 & $9.8 \%$ & $26.69[7.75,91.90]$ & & & & \\
\hline 2013 Munfus (2) & 17 & 26 & 9 & 26 & $10.4 \%$ & $3.57[1.14,11.19]$ & & & & \\
\hline 2013 Wang S. & 22 & 66 & 12 & 66 & $12.8 \%$ & $2.25[1.00,5.05]$ & & & & \\
\hline Total $(95 \% \mathrm{Cl})$ & & 656 & & 656 & $100.0 \%$ & $1.93[1.05,3.56]$ & & & & \\
\hline Total events & 275 & & 203 & & & & & & & \\
\hline $\begin{array}{l}\text { Heterogeneity: } \mathrm{Tau}^{2}= \\
\text { Test for overall effect }\end{array}$ & $\begin{array}{l}.59 ; \mathrm{Chi}^{2} \\
=2.12(\mathrm{P}\end{array}$ & $\begin{array}{l}37.06, \\
0.03)\end{array}$ & $d f=7(P$ & $<0.0$ & $001) ; 1^{2}=$ & & 0.01 & $\begin{array}{l}0.1 \\
\text { prechemo }\end{array}$ & postchemo & 100 \\
\hline
\end{tabular}

Figure 2. Meta-analysis of the effect of chemotherapy on EGFR mutation status in NSCLC patients.

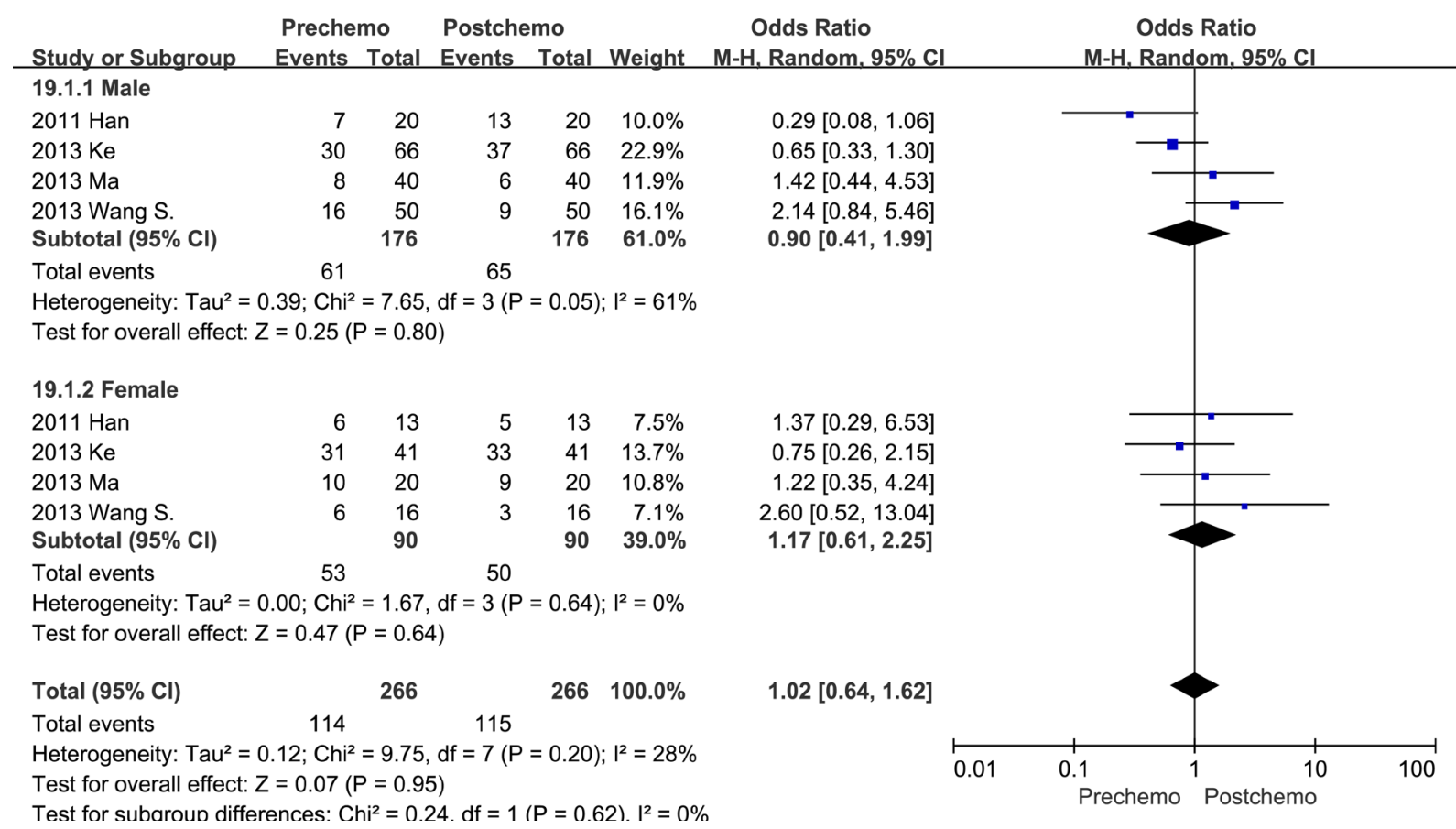

Figure 3. Meta-analysis of the effect of chemotherapy on EGFR mutation status based on gender. 


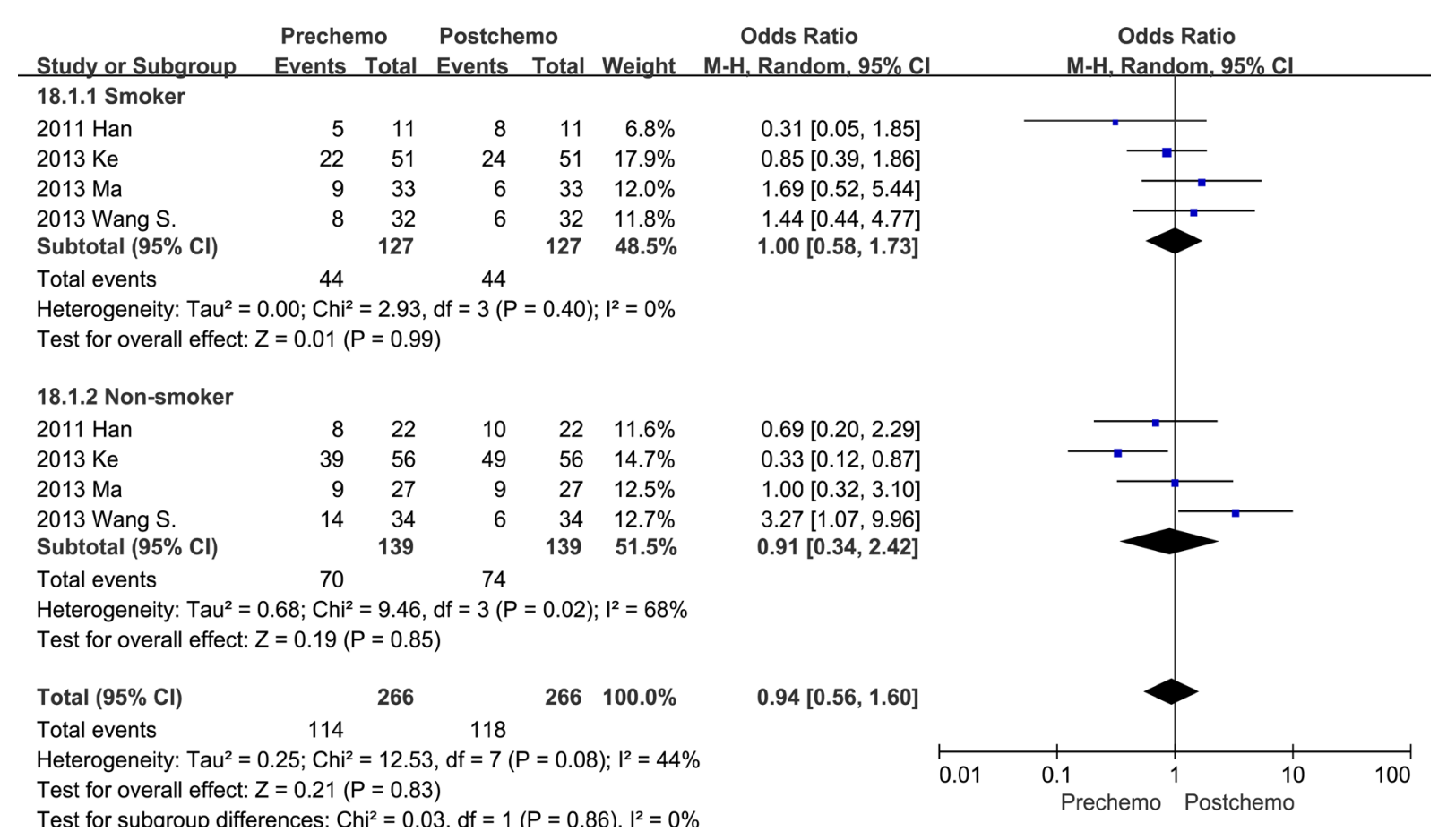

Figure 4. Meta analysis of the effect of chemotherapy on EGFR mutation status based on smoking history.

rapy on EGFR mutation alterations in males $(\mathrm{OR}=0.9,95 \% \mathrm{CI}: 0.41-1.99)$ and females $(\mathrm{OR}=1.17,95 \% \mathrm{CI}: 0.61-2.25)$ was not significantly different. We also observed no significant differences in EGFR status between smokers $(\mathrm{OR}=1.0$, 95\% CI: 0.58 - 1.73) and non-smokers (OR = 0.91, 95\% CI: $0.34-2.42)$. Both of these studies displayed no significant heterogeneity. Due to insufficient data, we were unable to meta-analyze other clinicopathological factors, such as age, tumor size, differentiation, and metastasis.

\subsection{Impact of Chemotherapy on EGFR Exon Loci}

Five studies contained data that could be used to examine the impact of chemotherapy on alterations in the EGFR exon 19 and 21 mutation status [9] [10] [11] [12] [13]. Statistical heterogeneity was found for this outcome. The total pooled OR of altered EGFR status for the exons was 1.83 (95\% CI $1.11-3.03$; p = 0.007). A subgroup analysis showed that in both the EGFR exon $19(\mathrm{OR}=2.11,95 \% \mathrm{CI}$ $0.98-4.58 ; \mathrm{p}=0.01)$ and the exon 21 mutation $(\mathrm{OR}=1.60,95 \%$ CI $0.78-3.27$; $=0.06$ ), chemotherapy was not an independent indicator of altered EGFR mutation status in NSCLC (Figure 5). A subgroup analysis of EGFR exon 18 and 20 mutations was not done due to insufficient data.

\subsection{Chemotherapy Response and EGFR Status}

Three studies included the relationship between chemotherapy outcome and EGFR mutation status (Figure 6). Theoretically, patients with EGFR mutations may have the advantage of a higher EGFR detection rate caused by a favorable chemotherapy response. In this subgroup analysis, beyond general postulations, 


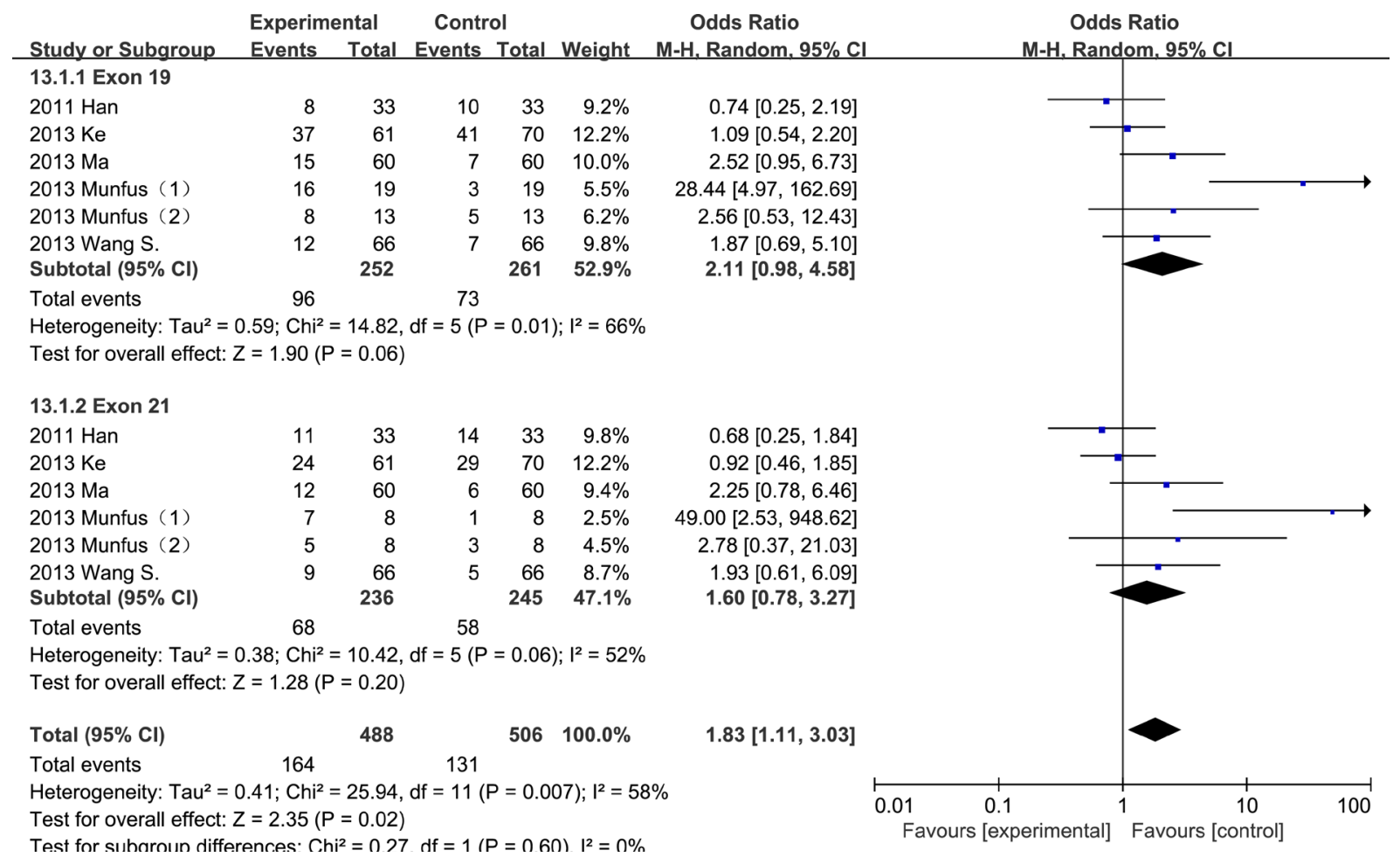

Figure 5. Meta-analysis of the effect of chemotherapy on EGFR mutation status in different EGFR exon loci.

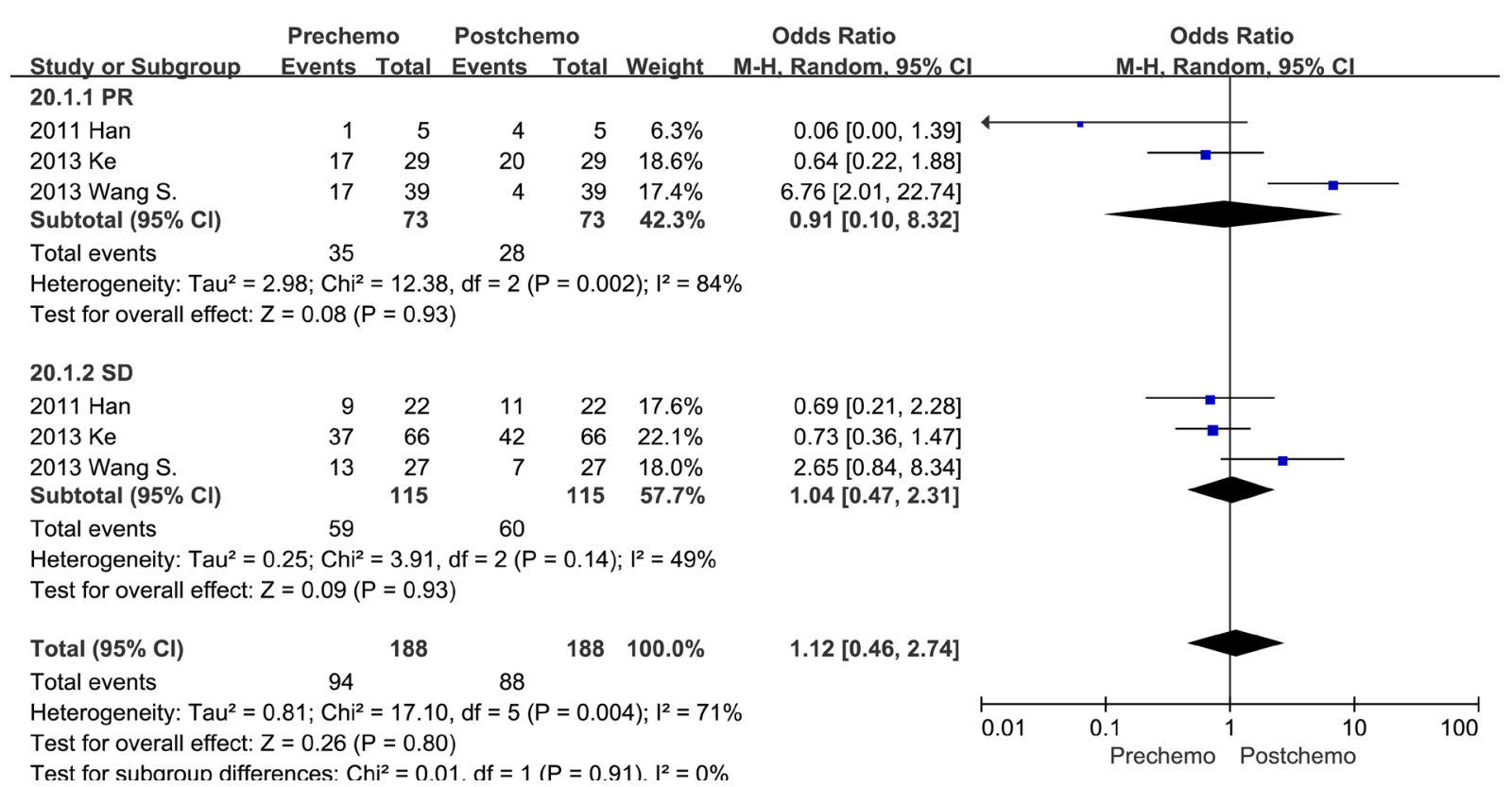

Figure 6. Meta-analysis of chemotherapy response on EGFR status.

there were no major differences between the partial-response population (PR: OR $0.91,95 \%$ CI: 0.10 - 8.32) and the stable-disease population (SD: OR = 1.04, $95 \%$ CI: 0.47 - 2.31). Subgroup analyses of the progressive disease (PD) and complete response (CR) populations were not done due to insufficient data. Of note, only three studies included this parameter, which was the least reliable item for the subgroup analysis. Large clinical trials may be necessary for a better 
understanding of the function of chemotherapy in EGFR mutation alterations in exon loci.

\subsection{Effect of Chemotherapy on EGFR Mutation Status Based on Determination Method}

The determination of EGFR status in free circulating DNA, as an alternative noninvasive approach to tissue biopsy, has been proposed in recent years. We separated studies with different detection methods for a subgroup analysis and found that the pooled OR value in the invasive-method subgroup was 2.96 (95\% CI: 1.01 - 8.65) (Figure 7). In the non-invasive-method subgroup, the OR value was 1.21 (95\% CI: 0.64 - 2.27). Therefore, the non-invasive method using blood was unable to detect altered EGFR mutations in our meta-analysis. This suggests that the use of blood for the evaluation of altered EGFR mutation status may not be as sensitive as methods using tissue.

\subsection{Publication Bias and Sensitivity Analyses}

With regard to publication bias, the funnel plots were largely symmetrical, suggesting no significant bias for all outcomes (Figure 8).

\section{Discussion}

Numerous recent studies have reported that patients with lung cancers harboring EGFR mutations can benefit from EGFR-TKIs, which are now established as first-line treatment options [14]. With the extensive application of EGFR-TKIs, different response rates have been observed between first-line EGFR-TKIs and

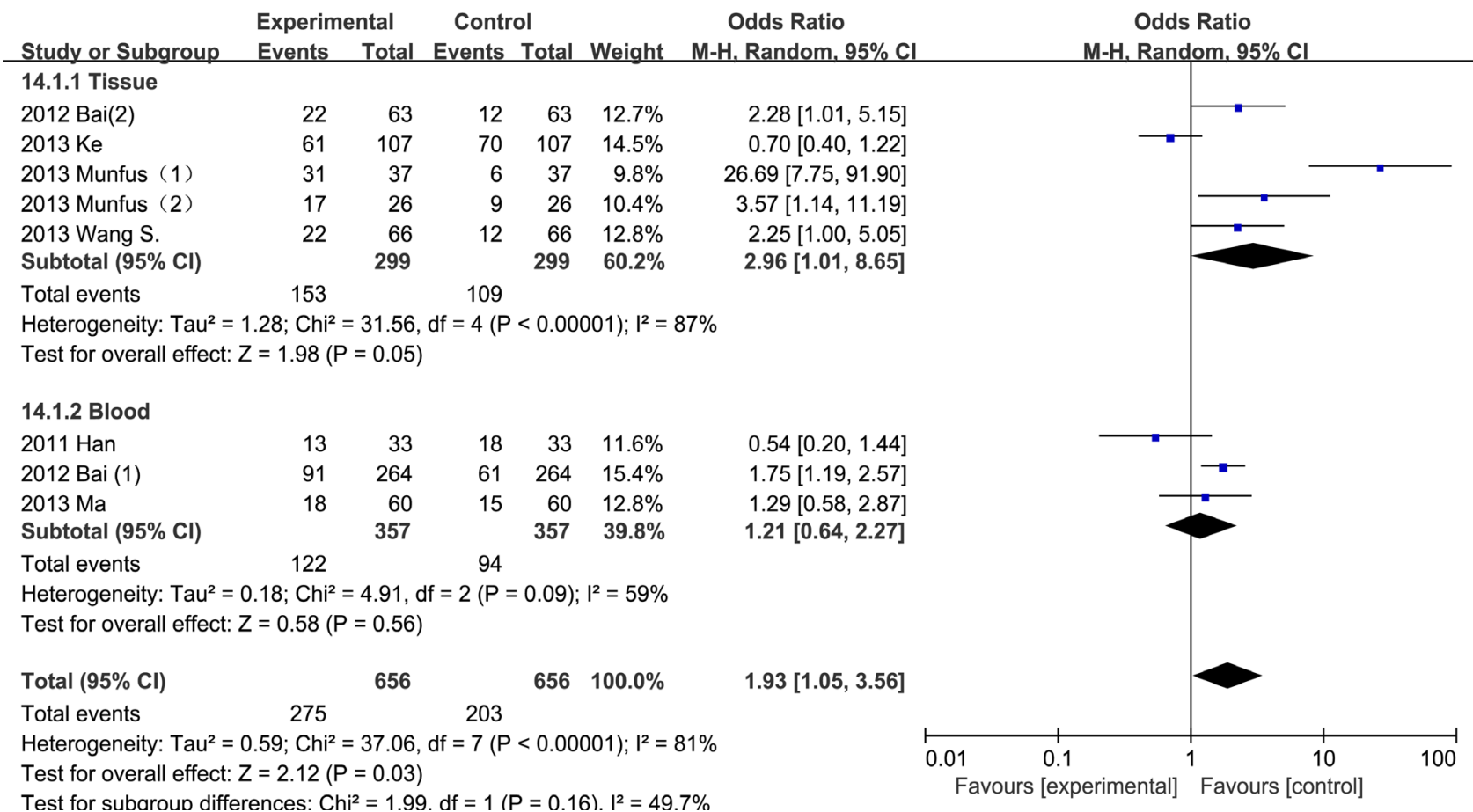

Figure 7. Meta-analysis of the effect of chemotherapy on EGFR mutation alterations based on different determination methods. 


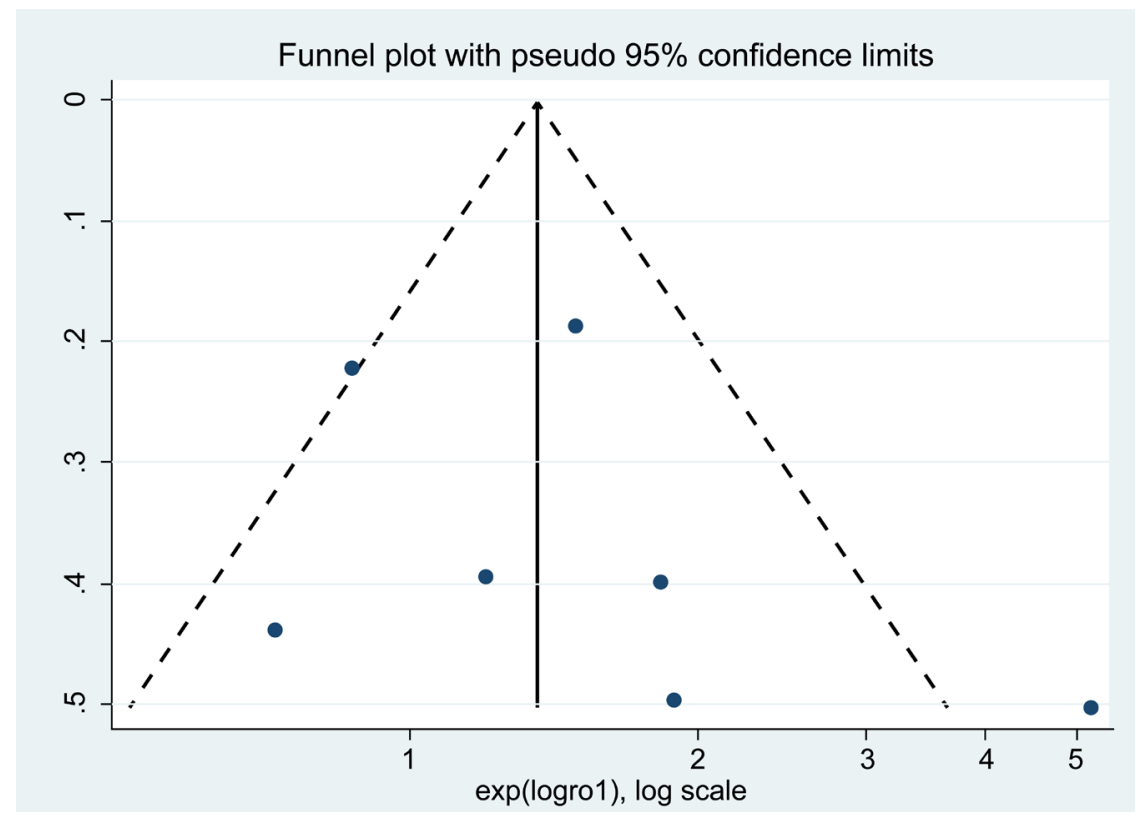

Figure 8. Funnel plot for all studies.

non-first-line EGFR-TKIs in patients with initial EGFR-positive mutations [5] [15]. In 2012, Honda et al. reported the disappearance of an activated EGFR mutation in a female patient after extensive treatment with cytotoxic drugs [7]. That same year, Bai et al. [8] proposed that the frequency of EGFR mutations might decrease after chemotherapy. In 2013, Shuhang et al. [13] reported a similar trend. In contrast, Delicia and colleagues [9] reported that the EGFR mutation rate increased from $12.8 \%$ to $27.3 \%$ after chemotherapy. Honggang and colleagues reported that EGFR mutation status may show an increasing trend [13]. Until now, no study has performed a meta-analysis to illustrate the potential role of chemotherapy in altering EGFR mutation status in NSCLC patients.

In clinical practice, exons 18 - 21 of the EGFR gene are analyzed by QPCR or sequencing. Due to insufficient data, we could not conduct a subgroup analysis for EGFR exon 18 and exon 20 mutations to determine the impact of chemotherapy on general EGFR loci; however, EGFR exon 19 deletions and exon 21 point mutations account for $90 \%$ of EGFR mutations [16] [17], which may help explain this practical problem. Analyses of the pooled data showed that platinumbased third-generation doublets did not alter exon 19 deletions (OR 2.11, 95\% CI $0.98-4.58 ; \mathrm{p}=0.01)$ or exon 21 point mutations (OR $=1.60,95 \%$ CI 0.78 3.27; $\mathrm{p}=0.06)$. However, chemotherapy exerted a positive effect on general EGFR mutation status in the NSCLC patients (OR $=1.93,95 \%$ CI $1.05-3.56 ; \mathrm{p}<$ 0.0001). This result signified that chemotherapy may be a risk factor for altered EGFR mutation status.

The determination of EGFR status prior to and after chemotherapy was limited to biopsy via fine needle aspiration, mediastinoscopy, fiber bronchoscopy, and surgical resection. Inconsistencies among sample specimens confound the accuracy of EGFR status, possibly due to the existence of intratumoral hetero- 
geneity [18] or sample insufficiency. Based on the hypothesis that circulating DNA in plasma may provide markers for lung cancer therapy, Sozzi et al. suggested the determination of EGFR status using free circulating DNA as an alternative, noninvasive approach [19]. Subsequently, a few large clinical trials have shown consistency among the tumor and serum samples, at a rate of approximately 79.7\% - 92.9\% [20] [21]. More recently, a meta-analysis performed by Chen reported that blood may be used as a substitute for tumor tissue in detecting EGFR mutations [22]. In the present study, we noted that the OR for the invasive-method group was 2.96 (95\% CI: $1.01-8.65)$, while the OR for the non-invasive-method group was 1.21 (95\% CI: $0.64-2.27$ ), which is insufficient to show altered EGFR status after chemotherapy. In terms of the trend of noninvasive gene mutation testing, identification of altered EGFR mutations using blood requires further validation in clinical practice.

In this analysis, chemotherapy's influence on EGFR mutation status was observed. Chemotherapy agents may alter the intratumoral natural selection that causes altered EGFR mutation status and activates the EGFR kinase pathway in different response groups. The underlying mechanism may be attributed to increased chemotherapy sensitivity in the EGFR-mutation population, thus offering a growth advantage for EGFR-non-mutation cells. However, in our analysis, the impact of chemotherapy on EGFR mutations in the partial-response group (PR: OR 0.91, 95\% CI: $0.10-8.32$ ) and the stable-disease group (SD: OR = 1.04, $95 \%$ CI: 0.47 - 2.31) did not show any significant differences.

The present study had several limitations. First, although the detection of EGFR mutations in plasma or serum has theoretical support [19] and there have been large-cohort clinical validations [20] [21] of the consistency of results obtained from biopsy, the use of serum ctDNA as guidance for TKI-targeted therapies is still being debated. In this meta-analysis, we analyzed only six studies including both detection methods, suggesting a risk of bias. Second, we could not perform subgroup analyses for different chemotherapy combinations. Irrespective of chemotherapy reagent combinations, the altered EGFR mutation status may vary with platinum-based third-generation doublets, which is worthy of further study. Third, the underlying mechanisms of altered EGFR mutation status could not be identified, as most of the included studies did not mention the biological impact of the EGFR pathway in the peri-chemotherapy period. Fourth, differences in the quality of the included articles might have led to bias in the meta-analysis. Finally, this meta-analysis only included studies published in the languages of English and Chinese.

\section{Conclusion}

Altered EGFR mutation status plays a crucial role in decision-making for EGFR-TKI therapies and subsequent second-line anti-cancer strategies in modern individualized treatment plans. In this meta-analysis, we attempted to include all studies that discussed pre- and post-chemotherapy EGFR status, in or- 
der to investigate the role of different chemotherapy agents in EGFR status alterations. We found that neoadjuvant or first-line chemotherapies may cause changes in EGFR mutation status in NSCLC patients regardless of gender, smoking history, EGFR loci, and response to chemotherapy. Interestingly, tumor tissue seems to be superior to blood for determining altered EGFR mutation status. Therefore, it may be necessary to obtain a second EGFR mutation status determination prior to second-line EGFR-TKI therapy and in patients with tumor recurrence following cytotoxic chemotherapy.

\section{Acknowledgements}

Professor Zhang and Dr. Shi made substantial contributions to the conception and design of the study, drafted the submitted article and revised it critically, provided final approval of the version to be published, and agreed to be accountable for all aspects of the work and ensuring that questions related to the accuracy or integrity of any part of the work are appropriately investigated and resolved.

Dr. Huang, Dr. Chen, Dr. Li and Ms. Wu made substantial contributions to the acquisition, analysis, and interpretation of the data, and critically revised the submitted article.

Dr. Ying Liang and Dr. Qipeng Zhou made substantial contributions to the data acquisition and article revisions.

Ms. Mo and Dr. Huang made substantial contributions to the statistical analysis.

This work was supported by the Youth Scientific Project Foundation of Guangzhou Medical University (2015A21). This work was partly supported by Science and Technology Planning Project of Guangdong Province, China (411234349027) and the Open Project Program of the State Key Laboratory of Respiratory Disease (2014SKLRD-O09).

\section{References}

[1] Siegel, R.L., Miller, K.D. and Jemal, A. (2015) Cancer Statistics, 2015. CA: A Cancer Journal for Clinicians, 65, 5-29. https://doi.org/10.3322/caac.21254

[2] Maemondo, M., Inoue, A., Kobayashi, K., Sugawara, S., Oizumi, S., Isobe, H., Gemma, A., Harada, M., Yoshizawa, H., Kinoshita, I., et al. (2010) Gefitinib or Chemotherapy for Non-small-cell Lung Cancer with Mutated EGFR. The New England Journal of Medicine, 362, 2380-2388. https://doi.org/10.1056/NEJMoa0909530

[3] Rosell, R., Carcereny, E., Gervais, R., Vergnenegre, A., Massuti, B., Felip, E., Palmero, R., Garcia-Gomez, R., Pallares, C., Sanchez, J.M., et al. (2012) Erlotinib Versus Standard Chemotherapy as First-line Treatment for European Patients with Advanced EGFR Mutation-positive Non-small-cell Lung Cancer (EURTAC): A Multicentre, Open-label, Randomised Phase 3 Trial. The Lancet Oncology, 13, 239-246. https://doi.org/10.1016/S1470-2045(11)70393-X

[4] Thatcher, N., Chang, A., Parikh, P., Rodrigues Pereira, J., Ciuleanu, T., von Pawel, J., Thongprasert, S., Tan, E.H., Pemberton, K., Archer, V., et al. (2005) Gefitinib 
Plus Best Supportive Care in Previously-treated Patients with Refractory Advanced Non-small-cell Lung Cancer: Results from a Randomised, Placebo-Controlled, Multicentre Study (Iressa Survival Evaluation in Lung Cancer). Lancet, 366, 1527-1537. https://doi.org/10.1016/S0140-6736(05)67625-8

[5] Mok, T.S., Wu, Y.L., Thongprasert, S., Yang, C.H., Chu, D.T., Saijo, N., Sunpaweravong, P., Han, B., Margono, B., Ichinose, Y., et al. (2009) Gefitinib or Carboplatin-Paclitaxel in Pulmonary Adenocarcinoma. The New England Journal of Medicine, 361, 947-957. https://doi.org/10.1056/NEJMoa0810699

[6] Mitsudomi, T., Morita, S., Yatabe, Y., Negoro, S., Okamoto, I., Tsurutani, J., Seto, T., Satouchi, M., Tada, H., Hirashima, T., et al. (2010) Gefitinib Versus Cisplatin Plus Docetaxel in Patients with Non-Small-Cell Lung Cancer Harbouring Mutations of the Epidermal Growth Factor Receptor (WJTOG3405): An Open Label, Randomised Phase 3 Trial. The Lancet Oncology, 11, 121-128.

https://doi.org/10.1016/S1470-2045(09)70364-X

[7] Honda, Y., Takigawa, N., Fushimi, S., Ochi, N., Kubo, T., Ozaki, S., Tanimoto, M. and Kiura, K. (2012) Disappearance of an Activated EGFR Mutation after Treatment with EGFR Tyrosine Kinase Inhibitors. Lung Cancer, 78, 121-124. https://doi.org/10.1016/j.lungcan.2012.07.003

[8] Bai, H., Wang, Z., Chen, K., Zhao, J., Lee, J.J., Wang, S., Zhou, Q., Zhuo, M., Mao, L., An, T., et al. (2012) Influence of Chemotherapy on EGFR Mutation Status Among Patients with Non-small-cell Lung Cancer. Journal of Clinical Oncology: Official Journal of the American Society of Clinical Oncology, 30, 3077-3083. https://doi.org/10.1200/JCO.2011.39.3744

[9] Munfus-McCray, D., Cui, M., Zhang, Z., Gabrielson, E., Askin, F. and Li, Q.K. (2013) Comparison of EGFR and KRAS Mutations in Primary and Unpaired Metastatic Lung Adenocarcinoma with Potential Chemotherapy Effect. Human Pathology, 44, 1286-1292. https://doi.org/10.1016/j.humpath.2012.10.016

[10] Han, R., Zhong, W., Zhao, J., Zhang, L., Xia, Y., Wang, H., Li, L. and Wang, M. Comparison of EGFR Mutation Status in Paired Pre- and Post-Chemotherapy Serum for advanced Pulmonary Adenocarcinoma. Chinese Journal of Lung Cancer (Zhongguo Fei Ai Za Zhi), 14, 127-131.

[11] Ke, H.G., Zhou, X.Y., Shen, Y., You, Q.S., Yan, Y. and Shen, Z.Y. (2012) The Relationship Between EGFR Gene Mutation Status and ERCC1 in Lung Adenocarcinoma of Chinese Patients Receiving Platinum-based Neoadjuvant Chemotherapy. Oncology Research, 20, 221-229. https://doi.org/10.3727/096504013X13589503482897

[12] Ma, Y. (2013) Change of EGFR Mutation Status in Pre- and Post-Chemotherapy Peripheral Blood for Advanced Non-Small-Cell Lung Cancer Patients. Shanxi Medical University.

[13] Wang, S., An, T., Duan, J., Zhang, L., Wu, M., Zhou, Q., Chen, J., Zhuo, M., Yang, L., Wang, Y., et al. (2013) Alterations in EGFR and Related Genes Following Neoadjuvant Chemotherapy in Chinese Patients with Non-Small-Cell Lung Cancer. PloS One, 8, e51021. https://doi.org/10.1371/journal.pone.0051021

[14] Tan, D.S., Yom, S.S., Tsao, M.S., Pass, H.I., Kelly, K., Peled, N., Yung, R.C., Wistuba, I.I., Yatabe, Y., Unger, M., et al. (2016) The International Association for the Study of Lung Cancer Consensus Statement on Optimizing Management of EGFR Mutation-positive Non-small-cell Lung Cancer: Status in 2016. Journal of Thoracic Oncology: Official Publication of the International Association for the Study of Lung Cancer, 11, 946-963.

[15] Rosell, R., Moran, T., Queralt, C., Porta, R., Cardenal, F., Camps, C., Majem, M., 
Lopez-Vivanco, G., Isla, D., Provencio, M., et al. (2009) Screening for Epidermal Growth Factor Receptor Mutations in Lung Cancer. The New England Journal of Medicine, 361, 958-967. https://doi.org/10.1056/NEJMoa0904554

[16] Lynch, T.J., Bell, D.W., Sordella, R., Gurubhagavatula, S., Okimoto, R.A., Brannigan, B.W., Harris, P.L., Haserlat, S.M., Supko, J.G., Haluska, F.G., et al. (2004) Activating Mutations in the Epidermal Growth Factor Receptor Underlying Responsiveness of Non-Small-Cell Lung Cancer to Gefitinib. The New England Journal of Medicine, 350, 2129-2139. https://doi.org/10.1056/NEJMoa040938

[17] Paez, J.G., Janne, P.A., Lee, J.C., Tracy, S., Greulich, H., Gabriel, S., Herman, P., Kaye, F.J., Lindeman, N., Boggon, T.J., et al. (2004) EGFR Mutations in Lung Cancer: Correlation with Clinical Response to Gefitinib Therapy. Science, 304, 1497-1500. https://doi.org/10.1126/science.1099314

[18] Gerlinger, M., Rowan, A.J., Horswell, S., Larkin, J., Endesfelder, D., Gronroos, E., Martinez, P., Matthews, N., Stewart, A., Tarpey, P., et al. (2012) Intratumor Heterogeneity and Branched Evolution Revealed by Multiregion Sequencing. The New England Journal of Medicine, 366, 883-892. https://doi.org/10.1056/NEJMoa1113205

[19] Sozzi, G., Conte, D., Leon, M., Ciricione, R., Roz, L., Ratcliffe, C., Roz, E., Cirenei, N., Bellomi, M., Pelosi, G., et al. (2003) Quantification of Free Circulating DNA as a Diagnostic Marker in Lung Cancer. Journal of Clinical Oncology: Official Journal of the American Society of Clinical Oncology, 21, 3902-3908.

https://doi.org/10.1200/JCO.2003.02.006

[20] Kimura, H., Suminoe, M., Kasahara, K., Sone, T., Araya, T., Tamori, S., Koizumi, F., Nishio, K., Miyamoto, K., Fujimura, M., et al. (2007) Evaluation of Epidermal Growth Factor Receptor Mutation Status in Serum DNA as a Predictor of Response to Gefitinib (IRESSA). British Journal of Cancer, 97, 778-784.

https://doi.org/10.1038/sj.bjc.6603949

[21] Qin, L., Zhong, W., Zhang, L., Li, L.Y. and Wang, M.Z. (2011) Comparison of Three Methods for Detecting Epidermal Growth Factor Receptor Mutations in Plasma DNA Samples of Chinese Patients with Advanced Non-Small-Cell Lung Cancer. Chinese Medical Journal, 124, 887-891.

[22] Mao, C., Yuan, J.Q., Yang, Z.Y., Fu, X.H., Wu, X.Y. and Tang, J.L. (2015) Blood as a Substitute for Tumor Tissue in Detecting EGFR Mutations for Guiding EGFR TKIs Treatment of Non-Small-Cell Lung Cancer: A Systematic Review and Meta-Analysis. Medicine, 94, e775. https://doi.org/10.1097/MD.0000000000000775 